\title{
A-to-I RNA editing alters less-conserved residues of highly conserved coding regions: Implications for dual functions in evolution
}

\author{
YUN YANG, ${ }^{1}$ JIANNING LV, ${ }^{1}$ BIN GUI, ${ }^{1}$ HENG YIN, ${ }^{1}$ XIAOJIE WU, ${ }^{1}$ YAOZHOU ZHANG, ${ }^{2}$ \\ and YONGFENG JIN ${ }^{1,2}$ \\ ${ }^{1}$ Institute of Biochemistry, College of Life Sciences, Zhejiang University, Hangzhou, Zhejiang, ZJ310058, People's Republic of China \\ ${ }^{2}$ Institute of Biochemistry, Zhejiang Sci-Tech University, Hangzhou, Zhejiang, ZJ 310018, People's Republic of China
}

\begin{abstract}
The molecular mechanism and physiological function of recoding by A-to-I RNA editing is well known, but its evolutionary significance remains a mystery. We analyzed the RNA editing of the $\mathrm{Kv} 2 \mathrm{~K}^{+}$channel from different insects spanning more than 300 million years of evolution: Drosophila melanogaster, Culex pipiens (Diptera), Pulex irritans (Siphonaptera), Bombyx mori (Lepidoptera), Tribolium castaneum (Coleoptera), Apis mellifera (Hymenoptera), Pediculus humanus (Phthiraptera), and Myzus persicae (Homoptera). RNA editing was detected across all Kv2 orthologs, representing the most highly conserved RNA editing event yet reported in invertebrates. Surprisingly, five of these editing sites were conserved in squid (Mollusca) and were possibly of independent origin, suggesting phylogenetic conservation of editing between mollusks and insects. Based on this result, we predicted and experimentally verified two novel A-to-I editing sites in squid synaptotagmin I transcript. In addition, comparative analysis indicated that RNA editing usually occurred within highly conserved coding regions, but mostly altered less-conserved coding positions of these regions. Moreover, more than half of these edited amino acids are genomically encoded in the orthologs of other species; an example of a conversion model of the nonconservative edited site is addressed. Therefore, these data imply that RNA editing might play dual roles in evolution by extending protein diversity and maintaining phylogenetic conservation.
\end{abstract}

Keywords: RNA editing; phylogenetic conservation; protein diversity; insect; squid

\section{INTRODUCTION}

The most common type of RNA editing involves the conversion of individual adenosine (A) bases to inosine (I) in RNA using adenosine deaminases acting on RNA (ADAR) (Bass 2002; Maas et al. 2003). The species-specific alteration of functionally important residues in a multitude of neuronal ion channels and presynaptic proteins through A-to-I RNA editing is profoundly important for the normal function of the nervous system (Higuchi et al. 2000; Palladino et al. 2000; Wang et al. 2000; Tonkin et al. 2002). Moreover, RNA editing could influence alternative splicing

Reprint requests to: Yongfeng Jin, Institute of Biochemistry, College of Life Sciences, Zhejiang University (Zijingang Campus), Hangzhou, Zhejiang, ZJ310058, People's Republic of China; e-mail: jinyf@zju.edu.cn; fax: 0086-571-88206478; or Yaozhou Zhang, Institute of Biochemistry, Zhejiang Sci-Tech University, Hangzhou, Zhejiang, ZJ 310018, P. R. China; e-mail: yaozhou@chinagene.com.

Article published online ahead of print. Article and publication date are at http://www.rnajournal.org/cgi/doi/10.1261/rna.1063708. decisions (Rueter et al. 1999; Maas et al. 2001; Bratt and Ohman 2003; Flomen et al. 2004; Laurencikiene et al. 2006; Jin et al. 2007; Schoft et al. 2007), antagonize RNA interference (RNAi) (Scadden and Smith 2001; Knight and Bass 2002; Tonkin and Bass 2003), modulate miRNA processing (Yang et al. 2006; Kawahara et al. 2007a), redirect miRNA silencing targets (Kawahara et al. 2007b), and create new exons (Lev-Maor et al. 2007). In addition, the widespread editing of Alu elements, which compose $\sim 10 \%$ of the human genome, plays a novel role in the primate lineage (Athanasiadis et al. 2004; Blow et al. 2004; Kim et al. 2004; Levanon et al. 2004). Overall, RNA editing plays an important role in regulating protein diversity and gene expression.

Despite progress made toward understanding the molecular mechanism and physiological function of A-to-I RNA editing, the challenge to verify its existence in evolution remains. In animals, A-to-I RNA editing mostly alters highly conserved or invariant coding positions in proteins 
(Reenan 2005), thus generating protein diversity. However, there are also many examples of A-to-I RNA editing at nonconservative protein positions (Patton et al. 1997; Grauso et al. 2002; Jin et al. 2007; Ohlson et al. 2007; Tian et al. 2008). Although the phylogenetic roles of nuclear RNA editing are interesting, our understanding may not be sufficient, possibly because previous studies have used small numbers of representative animals. Therefore, we investigated the RNA editing in a broad range of animals. The squid is poised to become an important model for understanding the biological importance of RNA editing, because two examined squid $\mathrm{K}^{+}$channels are heavily edited (for unknown reasons), and squid ADAR is extremely active in vitro (Patton et al. 1997; Rosenthal and Bezanilla 2002; Hoopengardner et al. 2003). We compared RNA editing between squid and insect Kv2 orthologs and found that five of these editing sites are conserved. This reveals phylogenetic conservation of editing between squid and insects. Based on these results, we predicted and experimentally verified two novel A-to-I editing sites in squid synaptotagmin I (sytI) transcript. A comparative analysis indicated that A-to-I RNA editing usually occurred in highly conserved coding regions, but mostly altered less-conserved coding positions of these regions. Moreover, more than half of these edited amino acids are genomically encoded in the orthologs of other species. Our results raise doubt about the hypothesis of Rosenthal and Bezanilla (2002) that extensive RNA editing in squid often leads to the introduction of amino acids with smaller side chains, as seen in antifreeze proteins of fish, which may increase flexibility of the molecule to compensate for cold because up to $80 \%$ of all A-to-I edits theoretically make smaller amino acids.

\section{RESULTS}

\section{Analysis of RNA editing in the insect $\mathrm{Kv} 2 \mathrm{~K}^{+}$channel}

Extensive A-to-I RNA editing sites were identified in the classical delayed rectifier $\mathrm{K}^{+}$channel $(S q K v 2)$ in the squid giant axon (Patton et al. 1997), one of which occurs in both Drosophila Kv2 (dShab) and mammalian Kv1.1 channels (Bhalla et al. 2004). To determine whether Kv2 orthologs were also edited in other insect species, we analyzed the sequences of RT-PCR amplification D. virilis is boxed. products to identify editing sites of three dipteran species, including Drosophila melanogaster, D. virilis, and one mosquito (Culex pipiens); the siphonapteran Pulex irritans; the lepidopteran Bombyx mori; the coleopteran Tribolium castaneum; the hymenopteran Apis mellifera; the phthirapteran Pediculus humanus; and the homopteran Myzus persicae. These sequence data allowed us to analyze the evolution of RNA editing over at least 300 million years.

A-to-I editing was detected across all Kv2 orthologs, but no two species possessed the same set of editing sites (Figs. $1 \mathrm{~A}, 2 \mathrm{~B}$ ). One common site (site 16) was frequently edited among all nine species of seven insect orders (Fig. 1A), mostly at a frequency of more than $50 \%$, representing the most highly conserved RNA editing site reported in invertebrates. Site 12 was conserved in D. melanogaster, C. pipiens, B. mori, and A. mellifera (Fig. 2B), but had a genomically encoded $\mathrm{G}$ in $P$. irritans and $P$. humanus. Site 14 was conserved across all insect species except B. mori

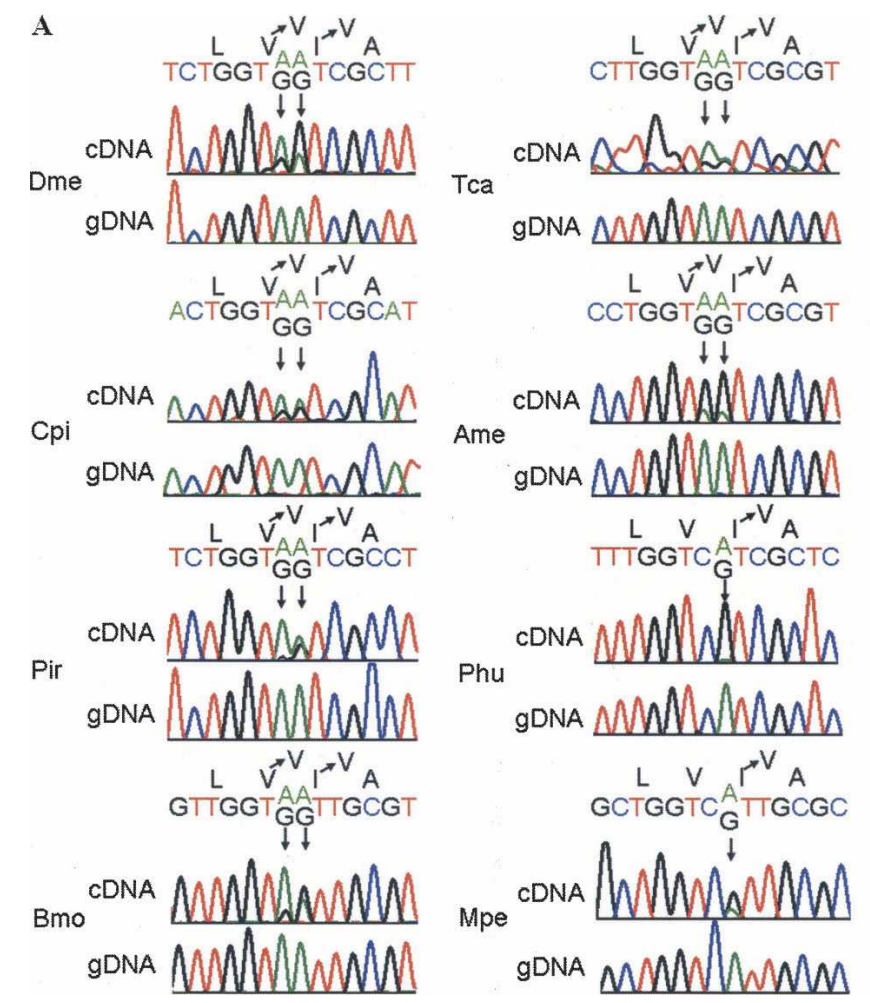

B

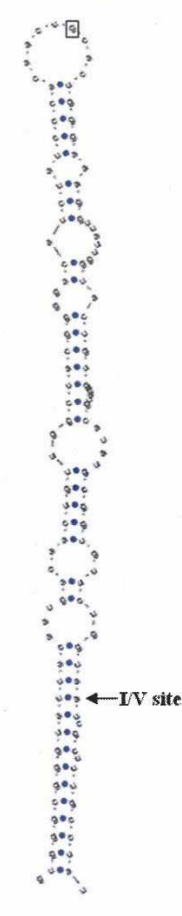

FIGURE 1. Editing in the Kv2 gene was conserved among all eight species of seven insect orders. (A) The editing sites (arrows) and their editing levels (A/G signal) among the Kv2 orthologs of D. melanogaster (Dme), C. pipiens (Cpi), P. irritans (Pir), B. mori (Bmo), T. castaneum (Tca), A. mellifera (Ame), P. humanus (Phu), and M. persicae (Mpe). RNA editing of the Kv2 genes of D. melanogaster have been described previously (Bhalla et al. 2004). The I/V editing site (arrows, right side of figure) is conserved among all eight species of seven insect orders. RNA editing in the synonymous site (arrows, left side of figure) is conserved among D. melanogaster, C. pipiens, P. irritans, B. mori, T. castaneum, and A. mellifera, while P. humanus and $M$. persicae are genomically incapable of A-to-I editing at the same location, as A is replaced by C. $(B)$ Prediction of secondary RNA structure for the edited exon of the D. melanogaster Kv2 transcript using the mFold program (Zuker 2003). The edited adenosine is marked by an arrow at the I/V site. The nucleotide in $D$. melanogaster that differs from 


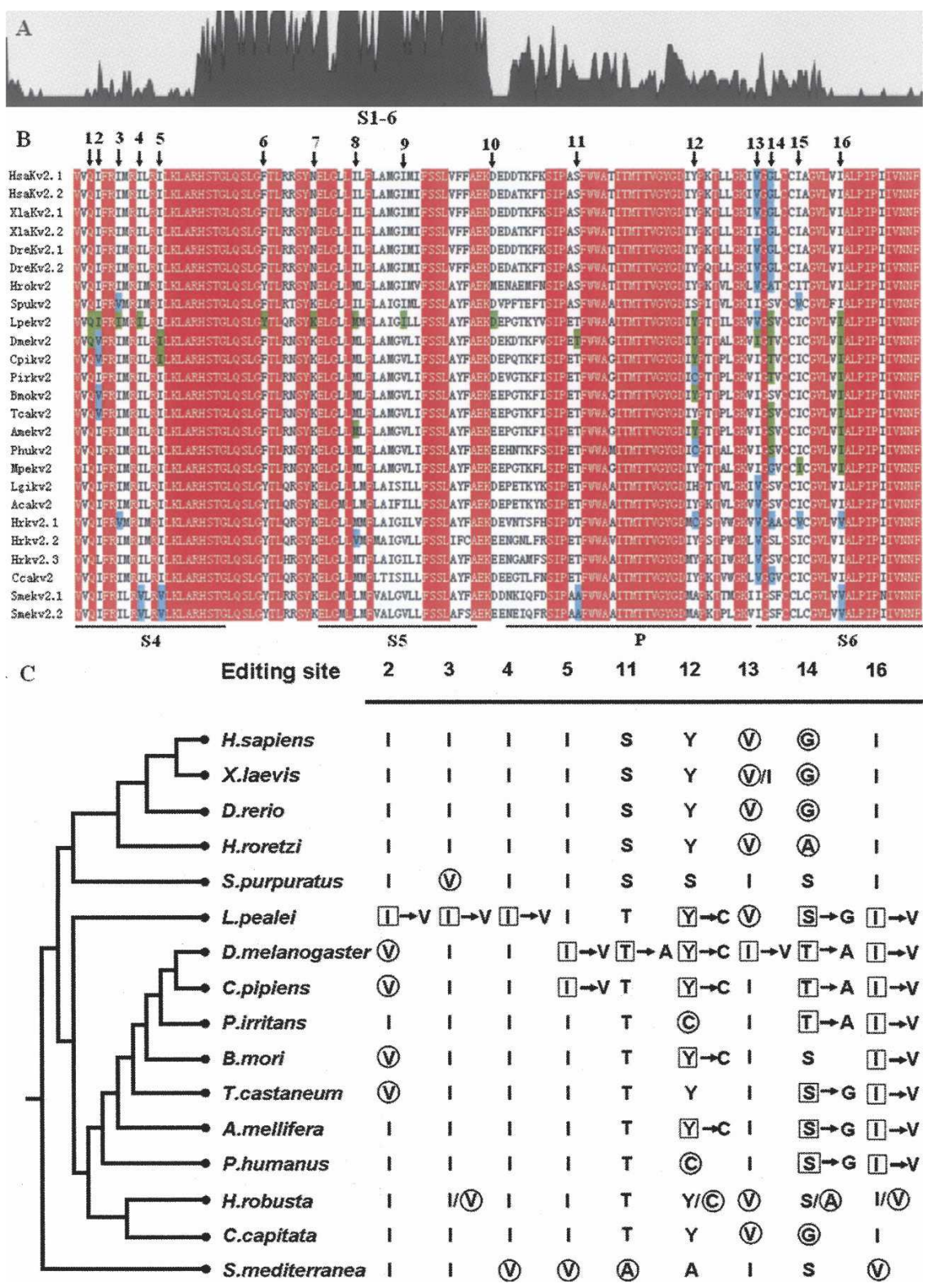

FIGURE 2. Phylogenetic conservation of A-to-I editing in the Kv2 $\mathrm{K}^{+}$channel of metazoans. (A) Conservation levels of the $\mathrm{Kv} 2 \mathrm{~K}^{+}$channel at the amino acid level using CLC Free Workbench 4. The high conservation level of the amino acid sequence suggests functional importance. (B) Comparison of amino acid sequences from various Kv2 orthologs encoding segment S4 through S6. Abbreviations: Hsa: Homo sapiens (AAA36156.1, NP_004761.2); Xla: Xenopus laevis (NP_001079257; NP_001079256); Dre: Danio rerio (XP_001332654.1; XP_001332827.1); Hro: H. roretzi (AB018545.1); Dme: D. melanogaster (NP_001036580.1); Cpi: C. pipiens (DS232049.1); Pir: P. irritans (EU616805); Bmo: B. mori (mb003438); Tca: T. castaneum; Ame: A. mellifera (XP_393546.3); Phu: P. humanus (DS235848.1); Mpe: M. persicae (EU616804); Lpe: L. pealei (CAA74748.1); Lgi: Lottia gigantea (Lotgi1/102260); Aca: Aplysia californica (AAC60504.2); Spu: S. purpuratus (XP_001179500); Hr: H. robusta (Helro1/119855, 94060, 108,240); Cca: Capitella capitata (Capca1/147057); Sme: S. mediterranea (AY066187; EG407084.1). RNA editing of the Kv2 $\mathrm{K}^{+}$channel genes from squid has been described previously (Patton et al. 1997). Amino acid residues showing complete conservation are shaded in red. Residues altered by RNA A-to-I editing are shaded in green, and genomic substitutions with edited residues at these positions are shaded in blue. (C) An evolutionary tree showing the phylogenetic relationships among these metazoan species. Phylogenetic tree data were adapted and modified from Sugden et al. (2003). Residues recoded by editing are enclosed in squares. Residues encoded genomically with edited residues are circled. and M. persicae (Fig. 2B), where the former was genomically incapable of Ato-I editing at the same location due to the lack of a first-position adenosine in its Ser codon (TCA) while the latter had a genomically encoded G. It is interesting to note that RNA editing occurred in the synonymous sites of D. melanogaster, C. pipiens, $P$. irritans, B. mori, T. castaneum, and A. mellifera (Fig. 1A), representing the most highly conserved RNA editing silent site yet reported in animals.

Because this editing site was so highly conserved, we analyzed secondary structures and an editing site complementary sequence (ECS). However, sequence alignment of the Kv2 genes failed to reveal regions of high sequence identity within upstream and downstream intronic regions surrounding the Ile/ Val editing site among 12 Drosophila species. Nevertheless, the exonic regions flanking the Ile/Val editing site are nearly identical, and are predicted by the program mFold (Zuker 2003) to form an extended secondary structure, conserved between D. melanogaster and D. virilis (Fig. 1B; Bhalla et al. 2004). The Kv2 genes of B. mori, T. castaneum, and A. mellifera share limited (70\%$76 \%$ ) nucleotide sequence similarity with D. melanogaster. However, their exonic regions of the Ile/Val editing site are also predicted to form base-paired structures pairing different coding sequences than those of Drosophila Kv2 (data not shown). In these cases, $\mathrm{Kv} 2$ genes are edited at the same Ile/Val site, possibly through different exondirected RNA secondary structures.

\section{Comparative analysis of RNA editing between insects and squid}

Insects phylogenetically belong to the class Insecta of the phylum Arthropoda, while squid is classified as a member of the Cephalopoda of the phylum Mollusca. Arthropoda and Mollusca diverged $\sim 550$ million years ago (Sugden et al. 2003). Analysis of Kv2 orthologs reveals a single copy in insects and squid, which seems to be ancestral and contains 17 editing sites occurring within a 360-nt 
segment in the Kv2 $\mathrm{K}^{+}$channel (Patton et al. 1997). Surprisingly, five of these sites were conserved between insects and squid, resulting in five amino acid conversions. To obtain insight into the evolution of Kv2 RNA editing, we examined RNA editing in the closely related phyla Arthropoda and Mollusca. RT-PCR analysis indicated that RNAediting sites of insect and squid Kv2 were not conserved in Anodonta woodiana (class Lamellibranchia; data not shown). Expressed sequence tag and cDNA analysis of Aplysia californica (Gastropoda), Lymnaea stagnalis (Crustacea), and Panulirus interruptus (Crustacea) (Baro et al. 1994) failed to provide evidence for RNA editing, suggesting that RNA editing events might occur independently in insects and squid. Alternatively, multiple independent loss and gain events could possibly result in the observed RNA editing.

\section{Prediction and verification of novel A-to-I editing sites}

We demonstrated that five editing sites were conserved for the Kv2 gene between the phyla Arthropoda (insect) and Mollusca (squid), suggesting a partial role of RNA editing in maintaining phylogenetic conservation. This information can be used to predict A-to-I editing sites by identifying positions where an RNA editing event would increase evolutionary conservation. A principal candidate gene was found in the squid synaptotagmin $I$ (sytI) transcript. As shown in Figure 3A, sequence alignment indicated two particular amino acids (Val334 and Ala350) in the published squid sytI cDNA sequence (accession numbers CAA51079 and BAA09866) (Bommert et al. 1993; Mikoshiba et al. 1995), because RNA editing results in substitution of Ile to $\mathrm{Val}$ at the V334 ortholog site in fruit flies and mosquitoes and in substitution of Thr to Ala at the A350 ortholog site in butterflies (Reenan 2005). Therefore, we predicted that Val334 and Ala350 might have been generated by RNA editing in squid. To test this prediction experimentally, we isolated total RNA from squid and compared the sequence to the genomic sequence. An adenosine was present in the genomic sequence at the position at which a $G$ and A mixed signal was present in the cDNA sequence (Fig. 3B). At these positions, the ACG (Thr) and AUC (Ile) codons were converted to ICG (Ala) and IUC (Val), respectively, because inosine is
A

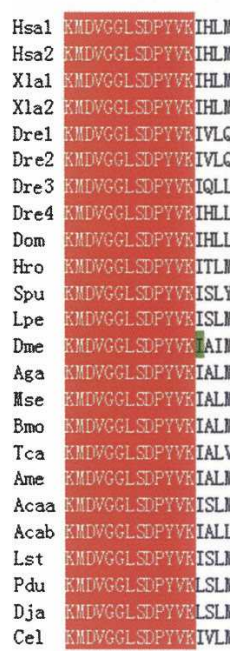

read as guanosine. It is assumed that sytI editing events in insects represent evolutionary novelties (Reenan 2005). Verification of these two A-to-I editing sites in squid in turn indicates that RNA editing events might occur independently in insects and squid.

\section{RNA editing usually alters less-conserved positions of highly conserved regions}

$\mathrm{Kv} 2 \mathrm{~K}^{+}$channels are evolutionarily ancient proteins conserved throughout the animal kingdom. We deduced the amino acid (AA) sequence of each Kv2 protein in Platyhelminthes, Annelida, Mollusca, Arthropoda, Echinodermata, and Chordata. Comparative analysis of the AA sequences revealed that there were several evolutionarily conserved sequences with the highest conservation in the S1-6 domain (Fig. 2A). The similarity of the AA sequences of any two species was $>70 \%$, and there were 73 absolutely conserved AA (Fig. 2B, shaded in red) within the S4-6 region (122 AA). The conservation in this region was

B
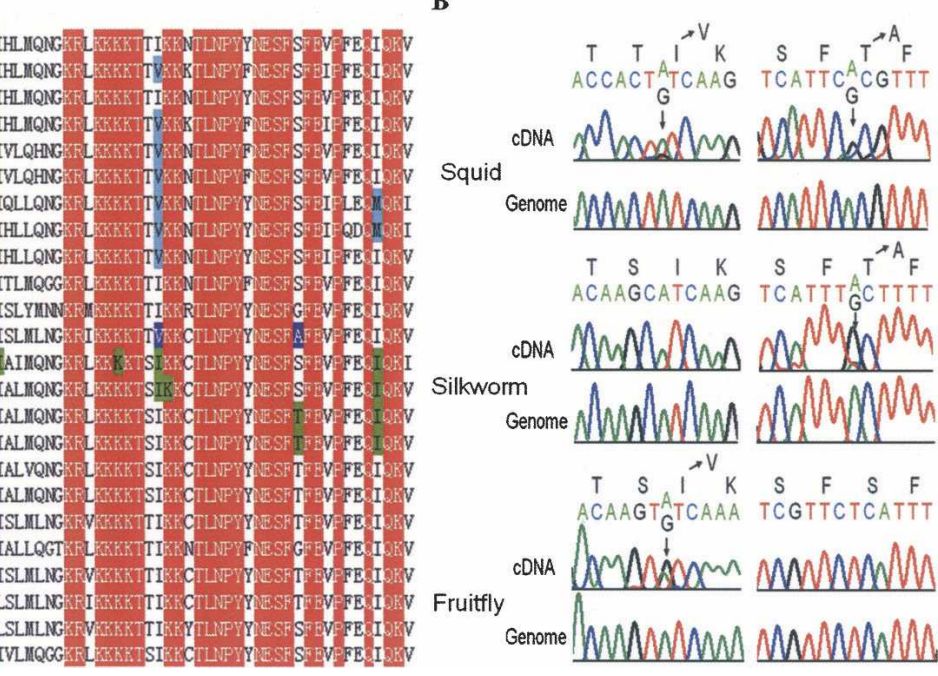

FIGURE 3. Two novel A-to-I editing sites were predicted and experimentally verified in squid sytI. (A) Comparison of amino acid sequences from various sytI orthologs. Abbreviations: Hsa: H. sapiens (AAA60609; BAC04354); Xla: X. laevis (CX838804, CX794956); Dre: D. rerio (NP_001018382, XM_683000.2; XM_691185.2); Lpe: L. pealei (CAA51079.1); Dme: D. melanogaster (AAF51205); Aga: A. gambiae (XP_317535); Bmo: B. mori (Scaffold005935); Tca: T. castaneum (XP_969399); Mse: Manduca sexta (AAK01129); Ame: A. mellifera (XP_392154); Aca: A. californica (AAQ91785; P41823); Lst: L. stagnalis (AAO83847); Dom: Discopyge ommata (AAA49228.1); Pdu: Platynereis dumerilii (ABR68850.1); Dja: Dugesia japonica (BAA85622); Spu: S. purpuratus (AAB67801.3); Hro: H. robusta (BAB18864); Cel: Caenorhabditis elegent (NP_001022129). RNA editing of the sytI in insects has been described previously (Reenan 2005). Amino acid residues showing complete conservation are shaded in red. Residues recoded by RNA editing are shaded in green, and genomic substitutions with edited residues at these positions are shaded in turquoise. Two candidate residues (shaded in dark blue) altered by RNA editing are predicted in squid. (B) Two editing sites in squid (Loligo chinensis) sytI are experimentally verified and compared to fruit fly (D. melanogaster) and silkworm (B. mori). RNA editing results in substitution of Ile to Val at the V334 ortholog site in the fruit fly, and in substitution of Thr to Ala at A350 ortholog site in the silkworm. Reverse transcriptase reactions were performed with oligo $(\mathrm{dT})_{15}$ on total RNA from squid. The RTPCR and PCR products were purified for direct sequencing. The editing sites (arrow) showed an $A / G$ mixed signal, while the DNA sequence exhibited only adenosine signals. 
markedly higher than in the other regions. Considering the fact that most editing sites were in this conserved region, it is tempting to think that A-to-I editing usually occurred in highly conserved coding regions.

However, RNA editing resulted in 16 AA changes in insects and squid (Patton et al. 1997), of which only one is completely conserved in 19 species, from low-level invertebrates (Schmidtea mediterranea) to vertebrates (human) (Fig. 2B). In this case, A-to-I editing at these sites mostly occurred at relatively less-conserved positions. An extensive analysis of known fruit fly editing genes also showed that editing occurred extensively at relatively less-conserved positions of the highly conserved region (Supplemental Fig. S1). The situation is similar in vertebrates, as the most intensively studied example of GluR-B RNA editing, the $\mathrm{Q} / \mathrm{R}$ site, is located at a relatively less-conserved position of the highly conserved transmembrane domain 2 (TM2) (Kung et al. 1996). The I/M editing site of the $\mathrm{GABA}_{\mathrm{A}}$ receptor subunit alpha3 is another example, as editing occurred at relatively less-conserved positions of the highly conserved TM3 (Ohlson et al. 2007). These data suggest that edited sites accumulate mutations much more rapidly than neighboring unedited sites. This idea is strengthened by recent analyses of RNA editing of nAChR alpha6, which suggested that edited sites accumulate mutations nearly twice as rapidly as unedited sites (Tian et al. 2008). These results contrast with previous reports in Drosophila, in which A-to-I RNA editing mostly altered highly conserved or invariant coding positions in proteins (Reenan 2005). However, these previous studies had limited sample sizes and compared closely related species. Our results indicate that, overall, RNA editing usually alters less-conserved positions of highly conserved regions, although some absolutely conserved residues are recoded by editing (Rosenthal and Bezanilla 2002; Reenan 2005; Jepson and Reenan 2007).

\section{Edited residues are frequently DNA encoded in other species}

We analyzed the amino acids at nonconservative editing positions. Interestingly, more than half of these edited amino acids were genomically encoded in the orthologs of other species (Fig. 2B,C). For example, editing results in the conversion of Ile to $\mathrm{Val}$ at site 2 in squid, but is genomically encoded in D. melanogaster, C. pipiens, B. mori, and $T$. castaneum (Fig. 2B,C). RNA editing at site 3 also recodes Ile to $\mathrm{Val}$ in squid, but is genomically encoded in Strongylocentrotus purpuratus and Helobdella robusta (Fig. 2B,C). Furthermore, editing at site 11 recodes Thr to Ala in the fruit fly and mosquito, but is genomically encoded in $S$. mediterranea (Fig. 2B,C). In addition, conversion of Ile to Val by RNA editing occurs at site 4 in squid and site 5 in fruit flies, but these are genomically encoded in S. mediterranea (Fig. 2B,C). A further A-to-I editing event results in substitution of Ile to $\mathrm{Val}$ at site 16 in squid and insects, but this is genomically encoded in $S$. mediterranea and H. robusta (Fig. 2B,C). These data suggest that RNA editing in squid and insects might help maintain phylogenetic conservation.

The possibility of phylogenetic conservation by RNA editing is strengthened by an analysis of squid $\mathrm{Kv1} \mathrm{K}^{+}$ channels, which are heavily edited (Rosenthal and Bezanilla 2002). Analysis of Kv1 orthologs from invertebrate and vertebrate species indicates that more than half of the edited amino acids in squid are genomically encoded in other species (Fig. 4A,B). A-to-I RNA editing results in extensive substitution of Ile to Val in squid, but this is genomically encoded in most vertebrate and invertebrate species (Fig. 4B). Analysis of Kv1 orthologs indicates that this subfamily mostly contains just a single member in most invertebrates, whereas an expanded Kv1 subfamily exists in vertebrates such as human and mouse (seven members), chicken (at least six members), and zebrafish (at least six members) (Fig. 4A). Further analysis of Kv1 orthologs reveals that Ile and Val are located at these ortholog sites in vertebrates. Considering their similar residues at these sites, it is tempting to speculate that the function of these residues is expanded by gene duplication and divergence in vertebrates; while squid and fruit flies employ RNA editing to expand their function, although their function might be affected by other residues (Jepson and Reenan 2007).

\section{An evolutionary example of a nonconservative edited site}

We found that one conserved editing site (Fig. 2B, site 14) is located in a nonconserved residue genomically encoded by Gly, Ser, Thr, and Ala in metazoans. Notably, according to amino acid structure and characteristics, Gly and Ser are similar to Thr and Ala, respectively (Fig. 5). Phylogenetic analysis in insects indicates that Ser represents the ancestral state at this site, at least before the radiation of Diptera and Lepidoptera (spanning $\sim 250$ million years). At the divergence of these two groups, Ser was converted to Thr in Diptera but remained Ser in Lepidoptera. Analysis of the orthologs of 12 Drosophila species (D. melanogaster, D. simulans, D. sechellia, D. yakuba, D. erecta, D. ananassae, D. pseudoobscura, D. persimilis, D. willistoni, D. mojavensis, D. virilis, and D. grimshawi) and three mosquito species (Anopheles gambiae, C. pipiens, Aedes aegypti) as well as the siphonapteran $P$. irritans showed that they possess a Thr at this site. Interestingly, this Kv2.1 homolog site has a genomically encoded Gly in all known vertebrates (Fig. 2B). Similar to squid (Patton et al. 1997), A-to-I RNA editing at this site results in substitution of Ser to Gly in T. castaneum, A. mellifera, P. humanus, which is genomically encoded in $M$. persicae and vertebrates (Fig. 2B). Conversely, editing at this site in fruit flies and mosquitoes recodes Thr to Ala, which is genomically encoded in Halocynthia roretzi and $H$. robusta (Fig. 2B,C). In summary, 

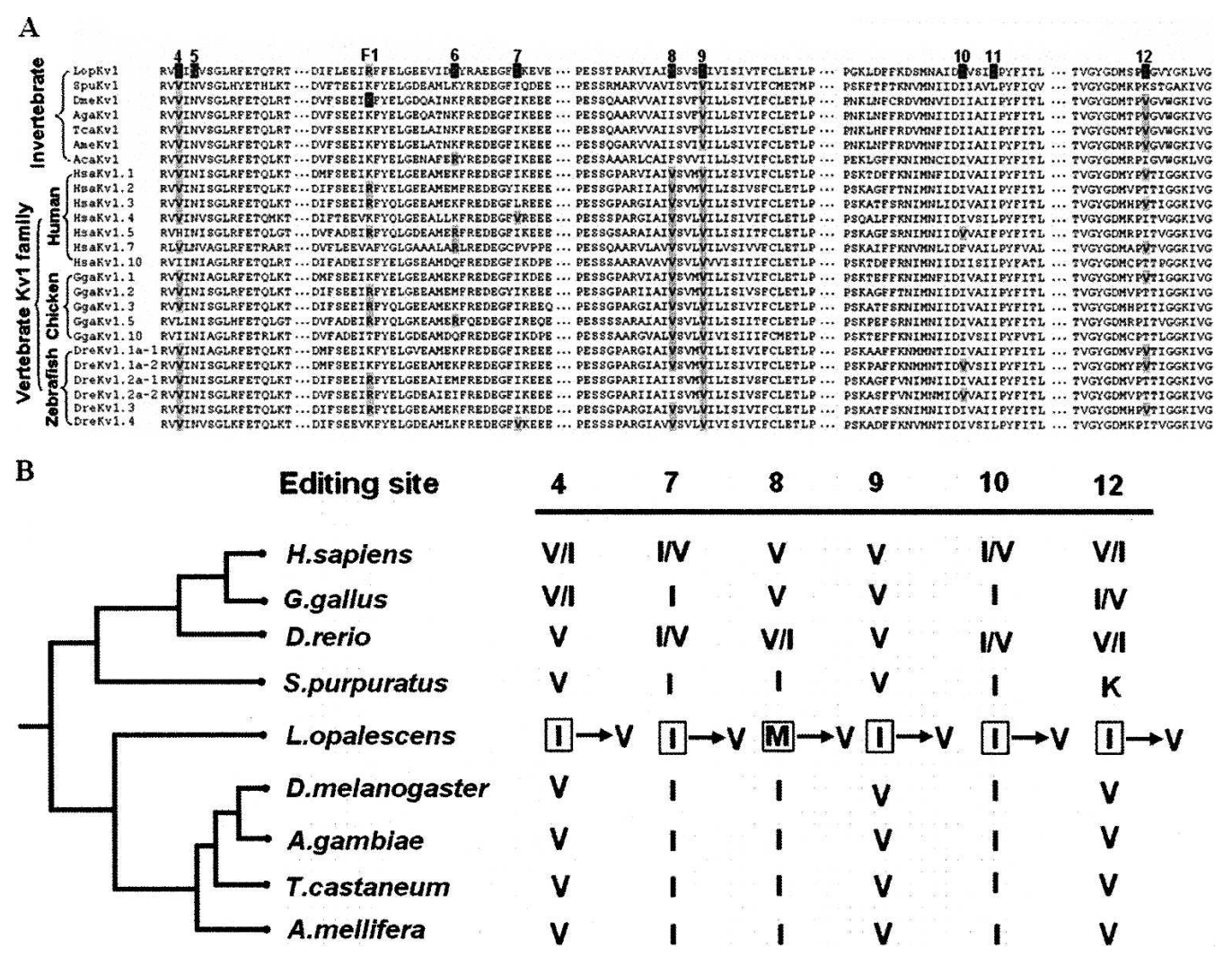

FIGURE 4. Phylogenetic conservation of A-to-I editing in the $\mathrm{Kv1} \mathrm{K}^{+}$channel of metazoans. $(A)$ Alignment of the amino acids of the Kv1 $\mathrm{K}^{+}$ channel genes. Abbreviations: Dme: D. melanogaster (NP_728123); Lop: L. opalescens (AAB02884); Spu: S. purpuratus (XP_001178924); Aga: A. gambiae (XP_310869); Tca: T. castaneum (XP_968511); Ame: A. mellifera (XP_391895); Aca: A. californica (AAA27756); Hsa: H. sapiens (NP_000208; BAF82750; NP_002223; NP_002224; NP_002225; NP_114092; NP_005540); Gga: Gallus gallus (XP_425660; NP_989794; NP_001025549; XP_417226; NP_989793); Dre: D. rerio (XP_687507; XP_001334455; XP_699869; NP_001104640; XP_695984; XP_687427). RNA editing of the $\mathrm{Kv1} \mathrm{K} \mathrm{K}^{+}$channel genes of D. melanogaster and squid has been described previously (Rosenthal and Bezanilla 2002; Hoopengardner et al. 2003). The residues altered by RNA editing sites are shaded in black, and genomic substitutions with edited residues at these positions are shaded in gray. $(B)$ An evolutionary tree showing the phylogenetic relationships among species. Residues recoded by RNA editing are enclosed in squares.

there are four alternative conversions for Ser (Fig. 5). In vertebrates, Ser was converted to Gly at the DNA level, while RNA editing recoded Ser into Gly in invertebrates such as squid, T. castaneum, A. mellifera, and P. humanus In the dipterans $D$. melanogaster and $C$. pipiens and the siphonapteran $P$. irritans, Ser evolved into Thr at the DNA level. Thr was then converted to Ala by subsequent RNA editing while Ser was converted to Ala at the DNA level in sea squirts ( $H$. roretzi). Interestingly, analysis of Kv2 orthologs reveals Ser and Ala at these equivalent positions in the $H$. robusta Kv2 family (Fig. $2 \mathrm{~B}$ ) and thus may resemble Ser and Gly generated by RNA editing in some insect species. This substitution by RNA editing provides an evolutionary model for extending protein diversity and maintaining phylogenetic conservation.

This conversion model of nonconservative edited sites also appears in other genes. For example, four residues (Gly, Ser, Thr, and Ala) are also located at the equivalent position of edited Thr350 in squid sytI (Nakhost et al. 2004). Based on the structural similarity of Ser and Thr and
Ala and Gly, switching these amino acids should not result in a major structural change while substitution between Ser/Thr and Ala/Gly could potentially result in a major functional change. For example, RNA editing generates a Ser/Gly substitution close to the fruit fly ADAR active site, which could result in less active ADAR than the genomeencoded, unedited isoform (Keegan et al. 2005). A Thr/Gly mutation at the equivalent position of sytI at the edited site shows greater resistance to proteolysis in the absence of calcium (Nakhost et al. 2004). Therefore, species-specific expansion at this site in DNA and RNA seems to have adaptive functional significance.

\section{DISCUSSION}

\section{The most highly conserved RNA editing event yet reported in invertebrates}

In invertebrates, most known RNA editing events are reported in Drosophila, and these editing sites are mostly 
Vertebrate DNA mutation
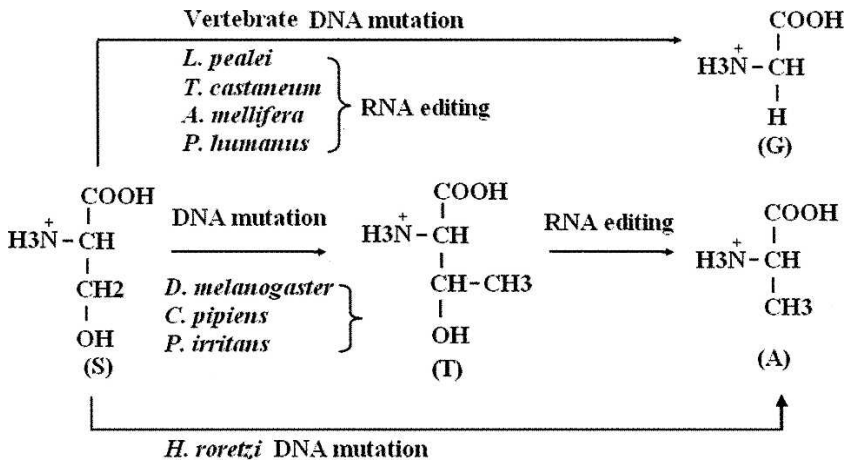

FIGURE 5. Conversion model of the nonconservative edited site (Fig. 2B, site 14) in Kv2 at the DNA and RNA level. Ser might represent the ancestral state at this position, and there are four alternative conversions for Ser. In most vertebrates, Ser was converted to Gly at the DNA level, while editing recoded Ser into Gly by RNA in invertebrates such as squid, T. castaneum, A. mellifera, and $P$. humanus. In the dipterans D. melanogaster and C. pipiens as well as the siphonapteran P. irritans, Ser was converted to Thr at the DNA level, and then to Ala by RNA editing, while Ser evolved into Ala at the DNA level in sea squirt $(H$. roretzi) and $H$. robusta. Notably, Gly and Ser are structurally similar to $\mathrm{Thr}$ and Ala, respectively.

confined to Diptera. In addition to Kv2, there are only two examples of RNA editing being conserved beyond Diptera. One example is sytI, for which fruit flies, dipteran mosquitoes, and butterflies (Lepidoptera) share one editing site, whereas honeybees and beetles do not edit sytI. Another example is nAChR alpha6, for which two editing sites are conserved in four insect orders, represented by D. melanogaster, B. mori, T. castaneum, and A. mellifera (Grauso et al. 2002; Jones et al. 2006; Jin et al. 2007), while mosquitoes do not edit nAChR alpha6 (Jones et al. 2005). In the present study, site 16 in Kv2 was conserved among seven orders, represented by D. melanogaster, P. irritans, B. mori, T. castaneum, A. mellifera, P. humanus, and M. persicae (Figs. 1A, 2B). RNA editing also occurred at this equivalent position in squid (Patton et al. 1997). Therefore, this represents the most highly conserved RNA editing event yet reported in invertebrates.

Conversion of Ile to Val at this site by RNA editing alters the rates of channel closure and inactivation in squid (Patton et al. 1997). In addition, the editing event (Ile/Val) profoundly affects channel inactivation conferred by accessory $\beta$-subunits at the equivalent position of human Kv1.1, while D. melanogaster Kv1 channels exhibit a similar effect through Ile/Val mutation (Bhalla et al. 2004). Interestingly, analysis of Kv2 orthologs revealed Ile as well as Val residues at these equivalent positions in the $H$. robusta Kv2 family (Fig. 2B,C), indicating that Ile-to-Val conversion occurs via gene duplication and divergence, thus mimicking this editing event. Therefore, it seems likely that Ile-to-Val conversion at this site has adaptive significance.

\section{Species-specific synonymous mutation potentially alters amino acids by RNA editing}

Site 14 in Kv2 was conserved in D. melanogaster, C. pipiens, $P$. irritans, T. castaneum, A. mellifera, and P. humanus but not B. mori (Fig. 2B,C). A similar situation occurred for nAChR alpha6, for which two editing sites were conserved in four orders, represented by D. melanogaster, B. mori, T. castaneum, and A. mellifera (Grauso et al. 2002; Jones et al. 2006; Jin et al. 2007), while editing was undetectable in dipteran mosquitoes. However, silkworm Kv2 lost this editing site while the two neighboring sites were edited. Thus, this codon might be located within a secondary structure, which would be necessary for the editing of the two neighboring sites. Because an ECS might form entirely from a conserved exonic sequence, it is very difficult to change ECS to escape editing. Therefore, codon synonymous mutation, which results in a lack of edited adenosine, might be a mechanism for escaping editing. This AGY-to-TCN synonymous substitution is also detected in other Lepidopteran insects, such as Epiphyas postvittana (EV813217) and Antheraea pernyi (data not shown). Editing in these species might be unnecessary and even deleterious, and thus AGY-to-TCN substitution can genomically escape this RNA editing. Further experiments are necessary to verify whether synonymous mutations from TCN to AGY can restore this RNA editing. Our observations imply that species-specific codon synonymous mutations might help regulate changes to amino acids via RNA editing.

\section{Implications for dual function of RNA editing in evolution}

Comparative analysis indicated that A-to-I editing usually occurred in highly conserved coding regions, but usually recoded less-conserved coding positions of these regions. Furthermore, more than half of these edited amino acids are genomically encoded in the orthologs of other species. These results suggest that RNA editing plays dual roles in evolution: extending protein diversity and maintaining phylogenetic conservation. Our analyses indicate that edited sites accumulate mutations much more rapidly than unedited sites, and thus evolution of sites undergoing mRNA editing is accelerated to enhance protein diversity. In addition, the RNA editing system is driven by genetic variation to maintain phylogenetic conservation, similar to plant mitochondrial RNA editing. In plants, RNA editing results in increased similarity with respect to homologous protein sequences among different organisms (Covello and Gray 1989; Gualberto et al. 1989; Tillich et al. 2005; Mulligan et al. 2007), suggesting that RNA editing helps repair otherwise deleterious genomic mutations (Gray and Covello 1993). Unlike most cases of plant RNA editing, however, animal genes are seldom fully edited. Much 
TABLE 1. Primers used for the RT-PCR and PCR analysis

\begin{tabular}{|c|c|c|}
\hline Species & Primer name & Primer sequence $\left(5^{\prime}-3^{\prime}\right)$ \\
\hline D. melanogaster & $\begin{array}{l}\text { Dmekv2-5-1 } \\
\text { Dmekv2-3-1 }\end{array}$ & $\begin{array}{l}\text { AGAAGTATCTGTGGGAGCTCCTCGA } \\
\text { GATCCATGGACTTGGCGAAGGCATC }\end{array}$ \\
\hline D. virilis & $\begin{array}{l}\text { Dvikv2-5-1 } \\
\text { Dvikv2-3-1 }\end{array}$ & $\begin{array}{l}\text { GGAGTTCGGCGAAGGTAAATGCGC } \\
\text { TGATATGATGGAAGGAGACAATGCT }\end{array}$ \\
\hline C. pipiens & $\begin{array}{l}\text { Cpkv2-5-1 } \\
\text { Cpkv2-5-2 } \\
\text { Cpkv2-3-1 } \\
\text { Cpkv2-3-2 }\end{array}$ & $\begin{array}{l}\text { AGCGATGACCTCGAGTACTGGGGCGT } \\
\text { CAGCGGGAGGAGGAAGAGTT } \\
\text { TTGATATGGTGGAAGCTGACGATGCT } \\
\text { GCCTTCTCGCGGCGCATCTG }\end{array}$ \\
\hline B. mori & $\begin{array}{l}\text { BmKv2-5-1 } \\
\text { BmKv2-3-1 } \\
\text { BmKv2-5-2 } \\
\text { BmKv2-3-2 }\end{array}$ & $\begin{array}{l}\text { TGGGACTTATTGGAGAAGCCGA } \\
\text { GTCCATGCTCTTGGCGAAAGCGT } \\
\text { GGGCACATGTGCTCAATATC } \\
\text { CTCGTAGGTTTATGTGGTGG }\end{array}$ \\
\hline T. castaneum & $\begin{array}{l}\text { TcaKv2-5-1 } \\
\text { TcaKv2-3-1 } \\
\text { TcaKv2-5-2 } \\
\text { TcaKv2-3-2 }\end{array}$ & $\begin{array}{l}\text { AGTGGCTCTGGGACATGCTCGAG } \\
\text { CAATGACGTCGATCAGGTCCATGCT } \\
\text { AACCGACCACCTCGATCGCG } \\
\text { CATCAACTTGAGGGACGCCTT }\end{array}$ \\
\hline A. mellifera & $\begin{array}{l}\text { AmeKv2-5-1 } \\
\text { AmeKv2-3-1 } \\
\text { AmeKv2-5-2 } \\
\text { AmeKv2-3-2 }\end{array}$ & $\begin{array}{l}\text { TCAGAAATGGCTATGGGATTTGCT } \\
\text { CGACGATGACATCGATGAGATCCAT } \\
\text { GAGAAACCAACTACGTCCATC } \\
\text { CGAAAGCGTCCCTCAGATTG }\end{array}$ \\
\hline P. humanus & $\begin{array}{l}\text { PhKv2-5-1 } \\
\text { PhKv2-5-2 } \\
\text { PhKv2-3-1 } \\
\text { PhKv2-3-2 }\end{array}$ & $\begin{array}{l}\text { TCTCGAATATTGGGGAGTCG } \\
\text { CGTGTTGTCAGCATAAGTACC } \\
\text { TGTGTCCACGATGACGTCAAT } \\
\text { TGCTCTTGGCGAAAGCGTCC }\end{array}$ \\
\hline Loligo chinensis & $\begin{array}{l}\text { LpeSyn-5-1 } \\
\text { LpeSyn-3-1 }\end{array}$ & $\begin{array}{l}\text { TATCGTTGATGCTTAACGG } \\
\text { CTGTATTTGTTCGAACGGGAC }\end{array}$ \\
\hline Degenerate primer & $\begin{array}{l}\text { DeKv2-5-1 } \\
\text { DeKv2-5-2 } \\
\text { DeKv2-3-1 } \\
\text { DeKv2-3-2 }\end{array}$ & $\begin{array}{l}\text { ATGGTNGARGCNGTNTGYATCACNTGGTT } \\
\text { AGTGCCTCRCCSGAYAARTGGAA } \\
\text { GTCCATRCTYTTSGCGAANGCRTC } \\
\text { CATCTGRTTYTTRTARAAYTCRGC }\end{array}$ \\
\hline
\end{tabular}

AIA/G(R) conversions. A model of the evolutionary mechanism of nuclear Ato-I RNA editing as an evolutionary intermediate of genetic variation was proposed (Tian et al. 2008). According to this model, Val-to-Ile mutation in sites 4, 9, and 12 of Kv1 might occur in squid (Fig. 4B) and subsequent A-to-I RNA editing at the homologous site may counteract this conversion, thus maintaining similarity at the protein level. Ile-to-Val mutation in sites 7, 8, and 10 of Kv1 might occur in vertebrates, mimicking RNA editing at these sites in squid (Fig. 4B). Although single editing events may often produce a protein that is not simply intermediate in character between fully edited and unedited proteins (Jepson and Reenan 2007), there are some examples of DNA mutation antagonizing RNA editing (Higuchi et al. 2000; Bhalla et al. 2004). Either way, RNA editing is more tolerated at these positions, as these edited residues are most commonly found at the ortholog positions of other species. Therefore, extensive RNA editing in squid might help maintain phylogenetic conservation rather than produce smaller amino acids.

\section{MATERIALS AND METHODS}

\section{Materials}

evidence indicates that unedited RNA is biologically important (Vissel et al. 2001). It is possible that partial RNA editing may be sufficient to maintain phylogenetic conservation. If so, this would support our implications for the dual function of RNA editing in evolution.

Our results raise doubt about the hypothesis of Rosenthal and Bezanilla (2002) that extensive RNA editing in squid often leads to the introduction of amino acids with smaller side chains. However, this is not surprising, because up to $80 \%$ of all A-to-I edits theoretically make smaller amino acids. Three bases in a DNA or RNA sequence specify a single amino acid according to a standard genetic codon. There are 64 triplet codons, 37 of which include A. In theory, these codons may produce 61 new codons by Ato-I editing, 35 of which result in amino acid substitution. Comparative analysis indicates that $80 \%$ of the edits $(28 / 35)$ make smaller amino acids. Of the remaining 20\%, three substitutions $[\mathrm{ATA}(\mathrm{Ile}) \rightarrow \mathrm{ATI}(\mathrm{Met})$; CAT/C $(\mathrm{H}) \mathrm{CIT} / \mathrm{C}(\mathrm{R})]$ change the size very little. The four examples of size increase are $\mathrm{CAA} / \mathrm{G}(\mathrm{Q})$ to $\mathrm{CIA} / \mathrm{G}(\mathrm{R})$ and $\mathrm{AAA} / \mathrm{G}(\mathrm{K})$ to
Human head lice ( $P$. humanus) and fleas ( $P$. irritans) were collected from the countryside, Zhejiang Province, China. Green peach aphid (M. persicae) was donated from the Mingguang Feng laboratory, Zhejiang University. Other insect materials were obtained as previously reported (Jin et al. 2007; Tian et al. 2008). Total RNA was isolated using the RNeasy Mini Kit (Qiagen) according to the manufacturer's protocol. Genomic DNA was isolated using the Universal Genomic DNA Extraction Kit (TaKaRa). Plasmid DNA was purified using the Qiagen plasmid isolation kit.

\section{Identification and cloning of the Kv2 genes of insects}

The sequences of the $\mathrm{Kv} 2 \mathrm{~K}^{+}$channel gene of D. melanogaster were described previously (Butler et al. 1989). To identify putative genes encoding the $\mathrm{Kv} 2 \mathrm{~K}^{+}$channel, we screened the genomes of C. pipiens (mosquito; Diptera), B. mori (silkworm; Lepidoptera), T. castaneum (red flour beetle; Coleoptera), A. mellifera (honeybee; Hymenoptera), and P. humanus with D. melanogaster $\mathrm{Kv} 2 \mathrm{~K}^{+}$ channel using the BLAST algorithm. Candidate $\mathrm{Kv} 2 \mathrm{~K}^{+}$channels were identified based on their considerable sequence homology with D. melanogaster $\mathrm{Kv} 2 \mathrm{~K}^{+}$channels. For P. irritans, M. persicae, 
and A. woodiana, degenerate primers were necessary to amplify products (Table 1). Amplification products were cloned into the pGEM-T Easy vector for sequencing. The nucleotide and amino acid sequences from each species were aligned using Clustal W.

\section{Analysis of RNA editing}

Total RNA was isolated from adult insects using the RNeasy Mini Kit (Qiagen). RNA preparations were digested with RNase-free DNase I (TaKaRa) to ensure the absence of genomic DNA contamination. First-strand cDNA was synthesized using the SuperScript III First-Strand Synthesis System for RT-PCR (Invitrogen) following the manufacturer's protocol. RT-PCR using the proofreading pfu DNA polymerase (TaKaRa) was performed using the primer pairs for the Kv2 gene in different species. The primers used for amplification of RT-PCR and PCR products are shown in Table 1. RT-PCR amplicons were directly sequenced after gel purification. The genomic PCR amplification products were also directly sequenced to demonstrate that genomic products give a pure A signal at editing sites, ruling out polymorphism. In addition, the RT-PCR products were cloned into the pGEM-T Easy vector (Promega), and approximately $20 \mathrm{cDNA}$ clones were sequenced for every sample to determine relative A-to-G abundance.

\section{SUPPLEMENTAL DATA}

Supplemental material can be found at http://www.rnajournal.org.

\section{ACKNOWLEDGMENTS}

We acknowledge Julia Hosp for help in the writing of the manuscript. This work was partly supported by research grants from the National Natural Science Foundation of China (90508007, 30770469), and 863 Program (2006AA10A119) and the Program for New Century Excellent Talents in University (NCET-04-0531).

Received March 6, 2008; accepted April 16, 2008.

\section{REFERENCES}

Athanasiadis, A., Rich, A., and Maas, S. 2004. Widespread A-to-I RNA editing of Alu-containing mRNAs in the human transcriptome. PLoS Biol. 2: e391. doi: 10.1371/journal.pbio.0020391.

Baro, D.J., Cole, C.L., Zarrin, A.R., Hughes, S., and HarrisWarrick, R.M. 1994. Shab gene expression in identified neurons of the pyloric network in the lobster stomatogastric ganglion. Receptors Channels 2: 193-205.

Bass, B.L. 2002. RNA editing by adenosine deaminases that act on RNA. Annu. Rev. Biochem. 71: 817-846.

Bhalla, T., Rosenthal, J.J., Holmgren, M., and Reenan, R. 2004. Control of human potassium channel inactivation by editing of a small mRNA hairpin. Nat. Struct. Mol. Biol. 11: 950-956.

Blow, M., Futreal, P.A., Wooster, R., and Stratton, M.R. 2004. A survey of RNA editing in human brain. Genome Res. 14: 2379-2387.

Bommert, K., Charlton, M.P., DeBello, W.M., Chin, G.J., Betz, H., and Augustine, G.J. 1993. Inhibition of neurotransmitter release by C2-domain peptides implicates synaptotagmin in exocytosis. Nature 363: 163-165.

Bratt, E. and Ohman, M. 2003. Coordination of editing and splicing of glutamate receptor pre-mRNA. RNA 9: 309-318.
Butler, A., Wei, A.G., Baker, K., and Salkoff, L. 1989. A family of putative potassium channel genes in Drosophila. Science 243: 943947.

Covello, P.S. and Gray, M.W. 1989. RNA editing in plant mitochondria. Nature 341: 662-666.

Flomen, R., Knight, J., Sham, P., Kerwin, R., and Makoff, A. 2004. Evidence that RNA editing modulates splice site selection in the 5HT2C receptor gene. Nucleic Acids Res. 32: 2113-2122.

Grauso, M., Reenan, R.A., Culetto, E., and Sattelle, D.B. 2002. Novel putative nicotinic acetylcholine receptor subunit genes, D $\alpha 5, \mathrm{D} \alpha 6$ and D 27 , in Drosophila melanogaster identify a new and highly conserved target of adenosine deaminase acting on RNA-mediated A-to-I pre-mRNA editing. Genetics 160: 1519-1533.

Gray, M.W. and Covello, P.S. 1993. RNA editing in plant mitochondria and chloroplasts. FASEB J. 7: 64-71.

Gualberto, J.M., Lamattina, L., Bonnard, G., Weil, J.H., and Grienenberger, J.M. 1989. RNA editing in wheat mitochondria results in the conservation of protein sequences. Nature 341: 660662.

Higuchi, M., Maas, S., Single, F.N., Hartner, J., Rozov, A., Burnashev, N., Feldmeyer, D., Sprengel, R., and Seeburg, P.H. 2000. Point mutation in an AMPA receptor gene rescues lethality in mice deficient in the RNA-editing enzyme ADAR2. Nature 406: $78-81$.

Hoopengardner, B., Bhalla, T., Staber, C., and Reenan, R. 2003. Nervous system targets of RNA editing identified by comparative genomics. Science 301: 832-836.

Jepson, J.E. and Reenan, R.A. 2007. RNA editing in regulating gene expression in the brain. Biochim. Biophys. Acta (in press). doi: 10.1016/j.bbagrm.2007.11.009.

Jin, Y., Tian, N., Cao, J., Liang, J., Yang, Z., and Lv, J. 2007. RNA editing and alternative splicing of the insect nAChR subunit $\alpha 6$ transcript: Evolutionary conservation, divergence, and regulation. BMC Evol. Biol. 7: 98.

Jones, A.K., Grauso, M., and Sattelle, D.B. 2005. The nicotinic acetylcholine receptor gene family of the malaria mosquito, Anopheles gambiae. Genomics 85: 176-187.

Jones, A.K., Raymond-Delpech, V., Thany, S.H., Gauthier, M., and Sattelle, D.B. 2006. The nicotinic acetylcholine receptor gene family of the honey bee, Apis mellifera. Genome Res. 16: 14221430.

Kawahara, Y., Zinshteyn, B., Chendrimada, T.P., Shiekhattar, R., and Nishikura, K. 2007a. RNA editing of the microRNA-151 precursor blocks cleavage by the Dicer-TRBP complex. EMBO Rep. 8: 763769.

Kawahara, Y., Zinshteyn, B., Sethupathy, P., Iizasa, H., Hatzigeorgiou, A.G., and Nishikura, K. 2007b. Redirection of silencing targets by adenosine-to-inosine editing of miRNAs. Science 315: 1137-1140.

Keegan, L.P., Brindle, J., Gallo, A., Leroy, A., Reenan, R.A., and O'Connell, M.A. 2005. Tuning of RNA editing by ADAR is required in Drosophila. EMBO J. 24: 2183-2193.

Kim, D.D., Kim, T.T., Walsh, T., Kobayashi, Y., Matise, T.C., Buyske, S., and Gabriel, A. 2004. Widespread RNA editing of embedded alu elements in the human transcriptome. Genome Res. 14: 1719-1725.

Knight, S.W. and Bass, B.L. 2002. The role of RNA editing by ADARs in RNAi. Mol. Cell 10: 809-817.

Kung, S.S., Wu, Y.M., and Chow, W.Y. 1996. Characterization of two fish glutamate receptor cDNA molecules: Absence of RNA editing at the Q/R site. Brain Res. Mol. Brain Res. 35: 119130.

Laurencikiene, J., Kallman, A.M., Fong, N., Bentley, D.L., and Ohman, M. 2006. RNA editing and alternative splicing: The importance of co-transcriptional coordination. EMBO Rep. 7: 303-307.

Lev-Maor, G., Sorek, R., Levanon, E.Y., Paz, N., Eisenberg, E., and Ast, G. 2007. RNA-editing-mediated exon evolution. Genome Biol. 8: R29. doi: 10.1186/gb-2007-8-2-r29. 
Levanon, E.Y., Eisenberg, E., Yelin, R., Nemzer, S., Hallegger, M., Shemesh, R., Fligelman, Z.Y., Shoshan, A., Pollock, S.R., Sztybel, D., et al. 2004. Systematic identification of abundant Ato-I editing sites in the human transcriptome. Nat. Biotechnol. 22: 1001-1005.

Maas, S., Patt, S., Schrey, M., and Rich, A. 2001. Underediting of glutamate receptor GluR-B mRNA in malignant gliomas. Proc. Natl. Acad. Sci. 98: 14687-14692.

Maas, S., Rich, A., and Nishikura, K. 2003. A-to-I RNA editing: Recent news and residual mysteries. J. Biol. Chem. 278: 1391-1394.

Mikoshiba, K., Fukuda, M., Moreira, J.E., Lewis, F.M., Sugimori, M., Niinobe, M., and Llinas, R. 1995. Role of the C2A domain of synaptotagmin in transmitter release as determined by specific antibody injection into the squid giant synapse preterminal. Proc. Natl. Acad. Sci. 92: 10703-10707.

Mulligan, R.M., Chang, K.L., and Chou, C.C. 2007. Computational analysis of RNA editing sites in plant mitochondrial genomes reveals similar information content and a sporadic distribution of editing sites. Mol. Biol. Evol. 24: 1971-1981.

Nakhost, A., Houeland, G., Blandford, V.E., Castellucci, V.F., and Sossin, W.S. 2004. Identification and characterization of a novel C2B splice variant of synaptotagmin I. J. Neurochem. 89: 354-363.

Ohlson, J., Pedersen, J.S., Haussler, D., and Ohman, M. 2007. Editing modifies the GABA(A) receptor subunit $\alpha 3$. RNA 13: 698-703.

Palladino, M.J., Keegan, L.P., O'Connell, M.A., and Reenan, R.A. 2000. A-to-I pre-mRNA editing in Drosophila is primarily involved in adult nervous system function and integrity. Cell 102: 437-449.

Patton, D.E., Silva, T., and Bezanilla, F. 1997. RNA editing generates a diverse array of transcripts encoding squid $\mathrm{Kv} 2 \mathrm{~K}^{+}$channels with altered functional properties. Neuron 19: 711-722.

Reenan, R.A. 2005. Molecular determinants and guided evolution of species-specific RNA editing. Nature 434: 409-413.

Rosenthal, J.J. and Bezanilla, F. 2002. Extensive editing of mRNAs for the squid delayed rectifier $\mathrm{K}^{+}$channel regulates subunit tetramerization. Neuron 34: 743-757.
Rueter, S.M., Dawson, T.R., and Emeson, R.B. 1999. Regulation of alternative splicing by RNA editing. Nature 399: 75-80.

Scadden, A.D. and Smith, C.W. 2001. RNAi is antagonized by $\mathrm{A} \rightarrow \mathrm{I}$ hyperediting. EMBO Rep. 2: 1107-1111.

Schoft, V.K., Schopoff, S., and Jantsch, M.F. 2007. Regulation of glutamate receptor B pre-mRNA splicing by RNA editing. Nucleic Acids Res. 35: 3723-3732.

Sugden, A.M., Jasny, B.R., Culotta, E., and Pennisi, E. 2003. Charting the evolutionary history of life. Science 300: 1691.

Tian, N., Wu, X., Zhang, Y., and Jin, Y. 2008. A-to-I editing sites are a genomically encoded G: Implications for the evolutionary significance and identification of novel editing sites. RNA 14: 211216.

Tillich, M., Funk, H.T., Schmitz-Linneweber, C., Poltnigg, P., Sabater, B., Martin, M., and Maier, R.M. 2005. Editing of plastid RNA in Arabidopsis thaliana ecotypes. Plant J. 43: 708-715.

Tonkin, L.A. and Bass, B.L. 2003. Mutations in RNAi rescue aberrant chemotaxis of ADAR mutants. Science 302: 1725.

Tonkin, L.A., Saccomanno, L., Morse, D.P., Brodigan, T., Krause, M., and Bass, B.L. 2002. RNA editing by ADARs is important for normal behavior in Caenorhabditis elegans. EMBO J. 21: 60256035.

Vissel, B., Royle, G.A., Christie, B.R., Schiffer, H.H., Ghetti, A., Tritto, T., Perez-Otano, I., Radcliffe, R.A., Seamans, J., Sejnowski, T., et al. 2001. The role of RNA editing of kainate receptors in synaptic plasticity and seizures. Neuron 29: 217227.

Wang, Q., Khillan, J., Gadue, P., and Nishikura, K. 2000. Requirement of the RNA editing deaminase ADAR1 gene for embryonic erythropoiesis. Science 290: 1765-1768.

Yang, W., Chendrimada, T.P., Wang, Q., Higuchi, M., Seeburg, P.H., Shiekhattar, R., and Nishikura, K. 2006. Modulation of microRNA processing and expression through RNA editing by ADAR deaminases. Nat. Struct. Mol. Biol. 13: 13-21.

Zuker, M. 2003. Mfold web server for nucleic acid folding and hybridization prediction. Nucleic Acids Res. 31: 3406-3415. 

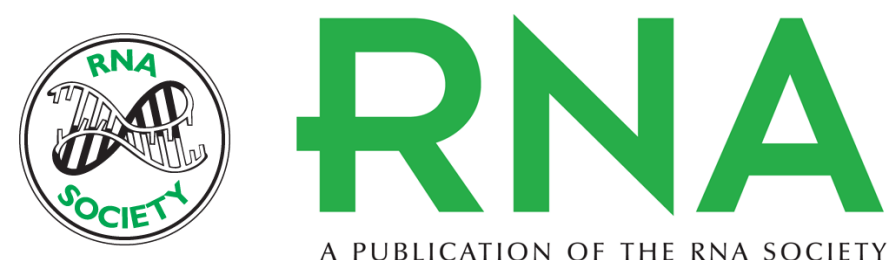

A PUBLICATION OF THE RNA SOCIETY

\section{A-to-I RNA editing alters less-conserved residues of highly conserved coding regions: Implications for dual functions in evolution}

Yun Yang, Jianning Lv, Bin Gui, et al.

RNA 2008 14: 1516-1525

Supplemental http://rnajournal.cshlp.org/content/suppl/2008/06/26/rna.1063708.DC1

Material

References This article cites 49 articles, 18 of which can be accessed free at:

http://rnajournal.cshlp.org/content/14/8/1516.full.html\#ref-list-1

License

Email Alerting Receive free email alerts when new articles cite this article - sign up in the box at the Service top right corner of the article or click here. 